\title{
Kinetics and yields of electron transfer in the inverted region
}

\author{
V. Gladkikh, A. I. Burshtein*, \\ Weizmann Institute of Science, Rehovot 76100, Israel \\ G. Angulo, \\ Graz University of Technology, Graz, Austria \\ Stéphane Pagès, Bernard Lang, Eric Vauthey \\ Department Physical Chemistry, University of Geneva, Geneva, Switherland
}

(May 21, 2004)

\section{SUPPORTING INFORMATION}

The rates of vibrational relaxation which accompanied the electron transfer should be proportional to the frequency of encounters between Pe and TCNE molecules exchanging vibrational quantum. This frequency was expected to be proportional to concentration of quenchers. This is actually the fact demonstrated in (Fig. 1S) with data borrowing from the Table I. The essential overlapping between the vibrational spectra of Pe and TCNE molecules is necessary for the quasi-resonance dipole-dipole energy transfer between them. As seen from Fig. 2S this is actually the fact well known from the literature. ${ }^{1,2}$

Figure 1S. Systematical dependence of $1 / \tau_{v}$ on quencher concentration $c$.

Figure 2S. Infrared spectra of perylene and TCNE taken from Refs. ${ }^{1,2}$

\footnotetext{
${ }^{1}$ Data from NIST Standard Reference Database 69 - March 2003. Release: NIST Chemistry WebBook.

${ }^{2}$ Langhoff, S. R. J. Phys. Chem. 1996, 1002819.
} 


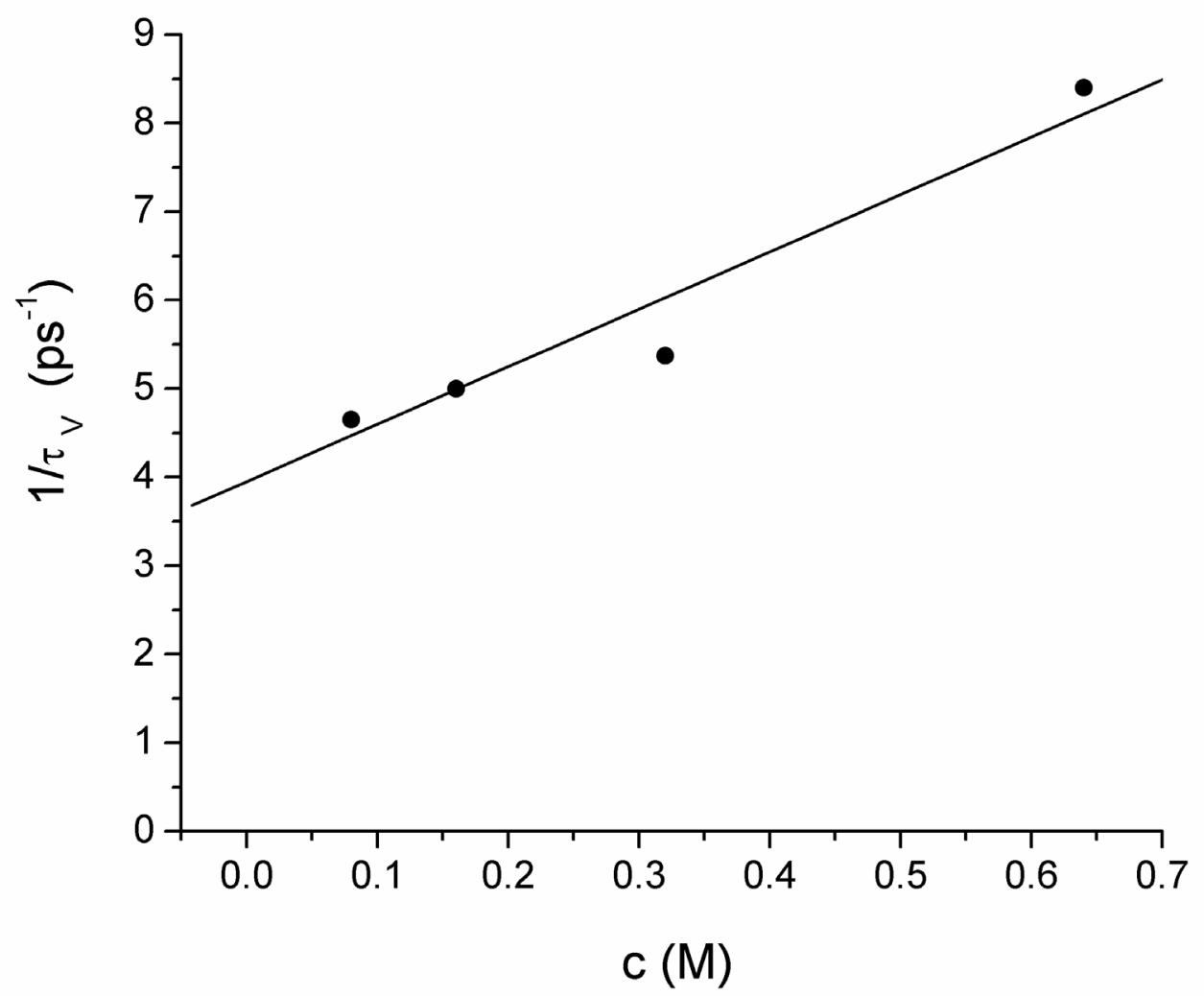




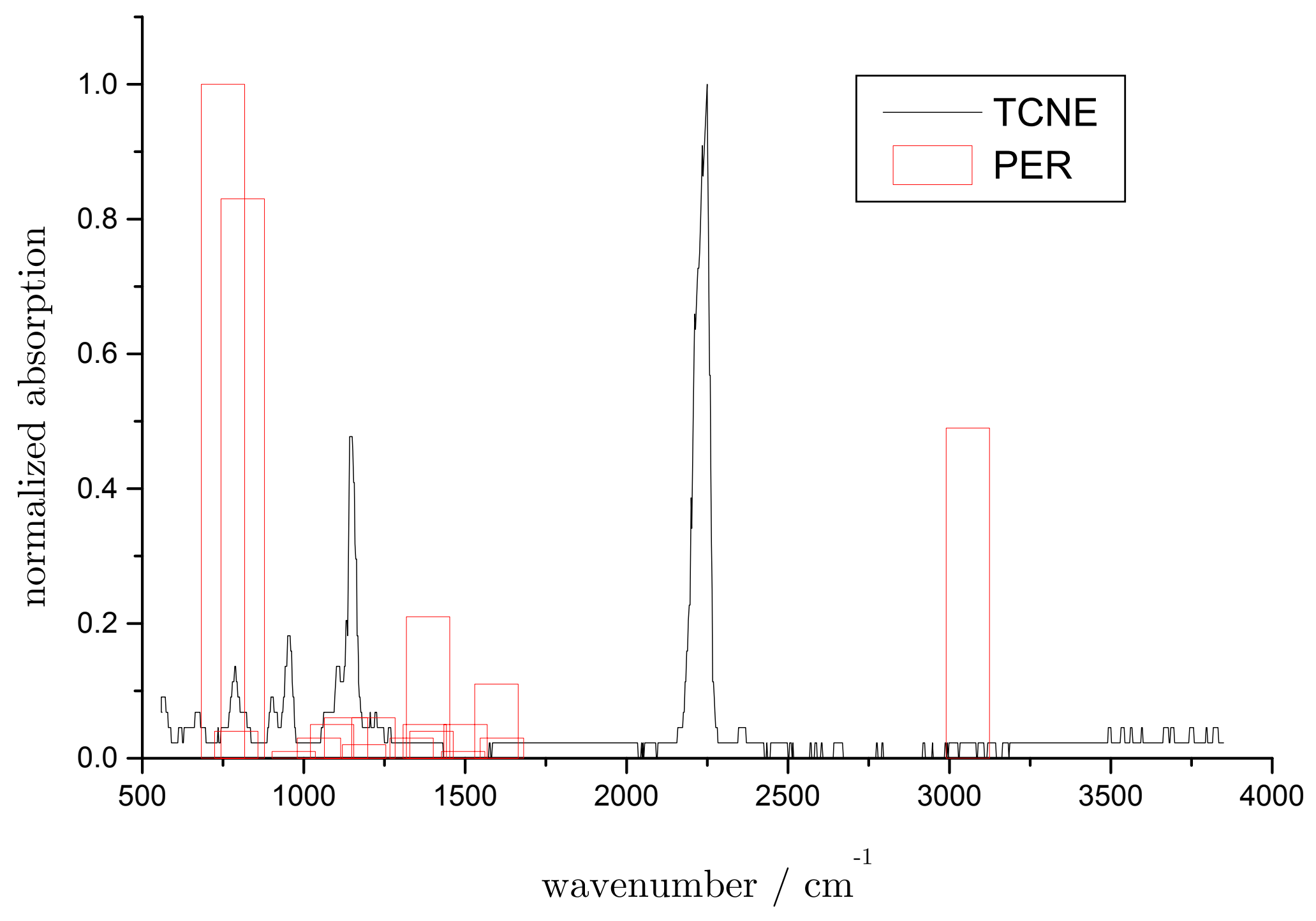

